Association for Information Systems AIS Electronic Library (AISeL)

Wirtschaftsinformatik Proceedings 2003

Wirtschaftsinformatik

September 2003

\title{
Multi-Channel Commerce: Hybridstrategien und Controlling
}

Karin Armbruster-Reif

Albert-Ludwigs-Universität Freiburg, karin.armbruster@vwl.uni-freiburg.de

Follow this and additional works at: http://aisel.aisnet.org/wi2003

\section{Recommended Citation}

Armbruster-Reif, Karin, "Multi-Channel Commerce: Hybridstrategien und Controlling" (2003). Wirtschaftsinformatik Proceedings 2003. 29.

http://aisel.aisnet.org/wi2003/29

This material is brought to you by the Wirtschaftsinformatik at AIS Electronic Library (AISeL). It has been accepted for inclusion in Wirtschaftsinformatik Proceedings 2003 by an authorized administrator of AIS Electronic Library (AISeL). For more information, please contact elibrary@aisnet.org. 
In: Uhr, Wolfgang, Esswein, Werner \& Schoop, Eric (Hg.) 2003. Wirtschaftsinformatik 2003: Medien - Märkte - Mobilität, 2 Bde. Heidelberg: Physica-Verlag

ISBN: 3-7908-0111-9 (Band 1)

ISBN: 3-7908-0116-X (Band 2)

(C) Physica-Verlag Heidelberg 2003 


\title{
Multi-Channel Commerce: Hybridstrategien und Controlling
}

\author{
Karin Armbruster-Reif \\ Albert-Ludwigs-Universität Freiburg
}

\begin{abstract}
Zusammenfassung: Nach dem Ende des Hype sind die Mehrzahl der Anbieter auf elektronischen Märkten im B2C-Bereich traditionelle Unternehmen. Sie können durch Vernetzung Synergien zwischen den bestehenden Vertriebskanälen und dem Internet nutzen und haben damit reinen Online-Händlern gegenüber Vorteile. Dennoch ist für die meisten dieser Unternehmen der Online-Kanal nicht rentabel. Der vorliegende Beitrag zeigt auf, dass die isolierte Betrachtung des OnlineKanals nicht ausreichend ist. Er erläutert die Vorteile der Vernetzung des Internets mit stationären Vertriebskanälen und stellt dar, wie diese Vorteile die Wirtschaftlichkeitsanalyse beeinflussen.
\end{abstract}

Schlüsselworte: Electronic Commerce, Hybridstrategien, Nutzeffektketten

\section{Aktuelle Situation im B2C-Electronic Commerce}

Das Internet hat sich in den letzten Jahren als neuer Vertriebskanal im Bereich Business-to-Consumer (B2C) etabliert. Aufgrund seiner Verbreitung und seiner besonderen Eigenschaften bietet es neue Möglichkeiten für Information, Kommunikation und Transaktionen.

Nachdem zu Beginn des Electronic Commerce-Hype hauptsächlich reine Online Händler im Mittelpunkt des Interesses standen, sind die meisten Anbieter im Internet heute traditionelle Anbieter. Sie können durch Vernetzung Synergien zwischen den bestehenden Vertriebskanälen und dem Internet nutzen und haben damit gegenüber reinen Online-Händlern Vorteile. Doch auch für viele traditionelle Unternehmen ist der Online-Kanal nicht rentabel. Für sie stellt sich die Frage, ob sie ihre Internet-Präsenz in Form eines Vertriebskanals aufrechterhalten oder das Angebot zukünftig auf Information und Kommunikation beschränken.

Um die Wirtschaftlichkeit des Online-Kanals sicherzustellen, müssen Unternehmen auch diesen Bereich in ihr Controlling einbeziehen. Dabei zeigt sich, dass eine isolierte Betrachtung des Online-Kanals nicht alle Erfolgsbeiträge dieses Kanals berücksichtigt. Vielmehr müssen auch die positiven Wirkungen, welche aus der Vernetzung des Internets mit den bestehenden Vertriebskanälen resultieren, 
berücksichtigt werden. Dies stellt für das Controlling eine neue Herausforderung dar. Die Tatsache, dass viele Unternehmen die kanalübergreifenden Wirkungen des Online-Kanals nicht berücksichtigen, macht deutlich, dass die erforderlichen Daten schwer zu gewinnen sind.

Im Folgenden werden zunächst die Möglichkeiten zur Vernetzung von traditionellen Vertriebskanälen und dem Internet dargestellt und die daraus resultierenden Vorteile erläutert. Im Anschluss wird die Wirtschaftlichkeit des Online-Kanals betrachtet. Hier folgen auf die Beschreibung der aktuellen Situation Überlegungen, welchen Einfluss die Berücksichtigung der kanalübergreifenden Wirkungen auf die Zukunft der Internet-Präsenz von Unternehmen hat. In einem Literaturüberblick werden verschiedene Ansätze für das Controlling des Electronic Commerce (E-Controlling) vorgestellt. Schließlich wird ein Ansatz zur Wirtschaftlichkeitsanalyse dargestellt, der die positiven Wirkungen des Online-Kanals auf andere Bereiche einbezieht.

\section{Hybridstrategien}

\subsection{Begriffsbestimmung}

Traditionelle Anbieter haben in den letzten Jahren das Internet als zusätzlichen Vertriebskanal in ihre Unternehmen integriert. Dabei handelt es sich um Unternehmen, die bisher den stationären Handel, den Versandhandel oder beides als Vertriebsweg genutzt haben. Diese Anbieter können entweder Hersteller oder Absatzmittler sein, wobei im Handel mit Konsumgütern Absatzmittler überwiegen. Allerdings haben viele Hersteller das Internet genutzt, um neben indirekten Vertriebskanälen erstmals auch einen direkten Vertriebskanal aufzubauen.

Vor dem Ausbau eines zusätzlichen Vertriebskanals im Internet, stellt sich für Unternehmen die Frage, ob und in wieweit sie den Online-Kanal in den existierenden Vertriebskanal-Mix integrieren. Hier bestehen zwischen den Extremformen Isolation und vollständige Integration zahlreiche Zwischenformen [GuGa00]. Betrachtet man die gewählten Integrationsformen von Unternehmen, so zeigt sich in den letzten Jahren ein Wandel [ArmSch02, S. 347]. In der Anfangszeit des Electronic Commerce wurde der Online-Kanal weitgehend von den bestehenden Vertriebskanälen isoliert. Mittlerweile hat sich gezeigt, dass der Electronic Commerce auch langfristig nur einen Teil des Konsumgütermarkts darstellen wird. Und so haben sich aufgrund des geringen Kaufvolumens nur wenige reine Online-Händler - wie beispielsweise Amazon - etablieren können. Dabei hat sich das mangelnde Vertrauen der Konsumenten als größtes Hindernis für den Kauf im Internet herausgestellt. Hier sind traditionelle Anbieter den reinen Online-Händlern gegenüber im Vorteil, weil ihnen von den Kunden mehr Vertrauen entgegengebracht wird $\left[\mathrm{St}^{+} 99\right.$, S. 53]. Um diesen Vertrauensvorteil zu nutzen, haben die traditionellen 
Anbieter in den letzten Jahren zunehmend ihr Internet-Angebot in den bestehenden Vertriebskanal-Mix integriert.

Anbieter, die neben stationären Vertriebskanälen auch das Internet als Kanal nutzen, werden mit Begriffen wie „Multi-Channel“-, „bricks-and-clicks“- oder „clicks-and-mortar"-Unternehmen bezeichnet [ArmSch02, S. 348]. Zur Unterscheidung von Unternehmen, die den Online-Kanal isoliert führen oder nur locker vernetzen, und Unternehmen, die ihre Kanäle weitgehend oder vollständig integrieren, werden im Folgenden die Begriffe Multikanalstrategie und Hybridstrategie verwendet.

Mit Multikanalstrategie werden alle Strategien bezeichnet, die das Angebot von mehr als einem Vertriebskanal bedeuten. Dagegen werden Strategien, die bestehende Vertriebskanäle mit dem Vertriebskanal Internet so vernetzen, dass die Kunden auch kanalübergreifende Kaufprozesse wählen können, als Hybridstrategien bezeichnet [z.B. Ro97, S. 186; StK199, S. 4]. Die Hybridstrategien bilden demnach eine Teilmenge der Multikanalstrategien.

Im Folgenden konzentriert sich die Betrachtung auf Unternehmen, die ursprünglich nur über einen oder mehrere stationäre Vertriebskanäle verfügten und später das Internet als zusätzlichen Vertriebskanal vollständig integriert haben. Dabei gibt es keine Segmentierung und jeder Vertriebskanal steht für alle Kundengruppen offen. Infolgedessen haben die Kunden in jeder Phase des Kaufprozesses die Möglichkeit, entweder den stationären Handel, den Online-Kanal oder auch beide Vertriebswege zu nutzen. Um die von den Kunden gewählten Kaufprozess darzustellen, wird eine Matrix verwendet, die zum einen die relevanten Phasen des Kaufprozesses Information, Vereinbarung, Abwicklung [La94, S. 18; SchmLi98] und After-Sales [GeSch99] enthält, zum anderen die verfügbaren Vertriebskanäle $\left[\mathrm{Schw}^{+} 02\right]$. Die Entscheidung, welche Vertriebskanäle in den einzelnen Phasen genutzt werden, liegt bei den Kunden $\left[\mathrm{Schw}^{+} 02\right]$. Somit bilden die Kunden erst durch die verkettete Nutzung der einzelnen Vertriebskanäle einen hybriden Kaufprozess. Hierbei sind viele unterschiedliche Kombinationen möglich. Ein möglicher Kaufprozess ist in Abbildung 1 dargestellt.

Die Abbildung zeigt beispielsweise den Kaufprozess für ein Paar Wanderschuhe. Der Kunde informiert sich zunächst im Online-Shop eines Anbieters (1). Nachdem er sich für ein Modell entschieden hat, geht er in eine stationäre Filiale des Anbieters, um die Schuhe anzuprobieren (2). Da der Anbieter Kundenkarteninhabern für den Kauf im Internet einen höheren Bonus gewährt, bestellt der Kunde die Wanderschuhe abends im Online-Shop (3). Er bezahlt die Ware gleich mit Kreditkarte. Als er zum Liefertermin nicht zu Hause ist, vereinbart er eine erneute Lieferung über das Internet (4). Über die richtige Pflege der Schuhe informiert er sich dann auf der Website des Anbieters (5). Als einer der Schuhe jedoch kurz danach schon die Sohle verliert, geht er damit zum Kundendienst der stationären Filiale (6). 


\begin{tabular}{|l|l|l|}
\hline Information & Stationärer Handel & Online-Shop \\
\hline Vereinbarung & & \\
\hline Abwicklung & & \\
\hline After-Sales & & \\
\hline
\end{tabular}

Abbildung 1: Beispiel für einen hybriden Kaufprozess

\subsection{Vorteile von Hybridstrategien}

Integrieren Unternehmen das Internet in ihren Vertriebskanal-Mix, entstehen Vorteile für alle beteiligten Vertriebskanäle. So profitiert sowohl der stationäre Handel vom Online-Kanal als auch der Online-Kanal von der stationären Präsenz des Unternehmens [Arm02, S. 4-8]. Weitere Vorteile wirken kanalübergreifend auf das gesamte Unternehmen. Eine erfolgreiche Internet-Präsenz verbessert beispielsweise das Image des Anbieters. Mit dem Angebot mehrerer Vertriebswege kann überdies auf unterschiedliche Vertriebskanalpräferenzen reagiert werden $\left[\mathrm{Ni}^{+} 01\right.$; SchoGo02]. Die kanalübergreifende Gewinnung von Kundeninformationen ermöglicht dem Unternehmen eine bessere Steuerung der einzelnen Vertriebskanäle und des Vertriebskanal-Mixes. Somit ermöglichen Hybridstrategien Unternehmen eine Verbesserung ihrer Marktposition besonders gegenüber Konkurrenten, die nur über traditionelle Vertriebskanäle oder ausschließlich über das Internet erreicht werden können [ArmSch02, S. 348-350].

\subsubsection{Vorteile für den Vertriebskanal Internet}

Mit dem Kauf im Internet verbinden Kunden ein höheres Risiko als mit dem Kauf in traditionellen Vertriebskanälen [TaTho00]. Die Verbindung eines Online-Shops zu einem bekannten Unternehmen reduziert aus Sicht der Kunden das Risiko des Online-Kaufs. Dabei kann die Risikoreduktion in eine partnerbezogene und eine transaktionsbezogene Komponente unterschieden werden [ArmSch02, S. 348349]. Die partnerbezogene Risikoreduktion bezieht sich auf die Tatsache, dass Kunden Anbietern im Internet, deren physische Präsenz sie kennen, mehr Vertrau- 
en entgegenbringen als reinen Online-Händlern [ $\mathrm{St}^{+} 99$ ]. Die transaktionsbezogene Risikoreduktion bezieht sich auf Risiken wie die Produktauswahl oder die Möglichkeit zum Umtausch. Hier bietet die stationäre Präsenz den Kunden die Gelegenheit, Produkte, deren Eigenschaften nur schwer medial vermittelbar sind (z. B. Parfums), vor dem erstmaligen Kauf persönlich zu begutachten [Ro97]. Auch bei Reklamationen ist der stationäre Handel Anlaufstelle für die Kunden.

Weitere Vorteile für den Online-Kanal sind die im Unternehmen vorhandene Markterfahrung, die Mitnutzung der bestehenden Infrastruktur und Einsparungen im Marketing. So profitiert der Online-Kanal beispielsweise von der Bekanntheit der traditionellen Vertriebskanäle und erlangt durch Werbung in diesen Kanälen Aufmerksamkeit.

Die beschriebenen Vorteile können Unternehmen jedoch nur nutzen, wenn Voraussetzungen wie eine gemeinsame Marke oder die Integration der Informationen, erfüllt sind [Goe02]. Zusätzlich müssen für Kanalkonflikte organisatorische Lösungen gefunden werden $\left[\mathrm{Bu}^{+} 97, \mathrm{~S} .41\right]$.

\subsubsection{Vorteile für die stationären Vertriebskanäle}

Traditionelle Anbieter können den Kunden der stationären Vertriebskanäle durch Zusatzleistungen im Internet einen verbesserten Service bieten und sich somit von ihren Konkurrenten differenzieren [St02]. In der Pre-Sales-Phase können die Kunden den Online-Shop als Informationsplattform nutzen. Im Vergleich zu den stationären Vertriebskanälen ermöglicht das Internet die Bereitstellung umfangreicherer Informationen, eine multimediale Darstellung und Hilfe durch Suchfunktionen und Produktvergleiche. Auch nach dem Kauf im stationären Geschäft können den Kunden im Internet Serviceleistungen geboten werden. Zum Beispiel der Download von Bedienungsanleitungen, FAQs oder Formulare für Anfragen. Durch das Internet können Anbieter zusätzlich ihr Produktangebot erweitern, indem sie im Online-Shop selten nachgefragte Produkte präsentieren oder individuelle Produkte im Rahmen von Mass Customization [Pi02] ermöglichen.

Nehmen die Kunden der stationären Vertriebskanäle den Pre-Sales- und AfterSales-Service im Online-Kanal in Anspruch, können im stationären Geschäft Einsparungen erzielt werden. Denn die Bereitstellung von Informationen im Internet ist kostengünstig und die Kunden übernehmen einen Teil der Aufgaben selbst indem sie zum Beispiel Formulare ausfüllen [St02]. Auch für das Marketing ergeben sich Einsparungen, da die durch das Internet gewonnen Kundendaten zielgerichtete Maßnahmen ermöglichen. Bei Werbemaßnahmen in traditionellen Medien ermöglicht der Hinweis auf die Internet-Präsenz eine kostengünstige Bereitstellung weiterer Informationen für Interessenten.

Weil heute die meisten Konsumenten das Internet nur zur Information nutzen und weiterhin im stationären Handel kaufen, können durch die Internet-Präsenz vor allem neue Kunden für das stationäre Geschäft gewonnen werden. Hierzu müssen 
Informationen über die Filialen auf der Website leicht zu finden sein. Eine Möglichkeit, die Zahl der gewonnenen Kunden zu erhöhen sind Gutscheine, die die Konsumenten ausdrucken und in den Filialen einlösen können.

Erhöht sich schließlich durch die zusätzliche Nachfrage im Online-Shop das Einkaufsvolumen des Anbieters bei seinen Lieferanten so sehr, dass ihm bessere Konditionen gewährt werden, so profitiert auch der traditionelle Vertriebskanal davon.

\section{Wirtschaftlichkeit des Vertriebskanals Internet}

\subsection{Aktuelle Situation}

Nach dem Electronic Commerce Hype hat sich mittlerweile Ernüchterung breit gemacht, da nur wenige Unternehmen mit ihrem Online-Shop Gewinne erzielen. Die Kosten eines Online-Shops sind im Vergleich zu einem stationären Vertriebskanal niedrig, werden aber häufig unterschätzt. Zum einen entstehen durch die Erstellung und Implementierung eines Online-Shops mit umfangreichen Funktionen und der erforderlichen Logistik-Infrastruktur [Kla00] hohe Planungs- und Investitionskosten. Zum anderen fallen durch das Online-Angebot regelmäßig Betriebskosten an [Bru97, S.127-128]. Ein Teil der Betriebskosten - zum Beispiel die Aktualisierung der Inhalte oder sicherheitsbedingte Updates der Software sind fixe Kosten. Die übrigen Betriebskosten - wie beispielsweise die Personalkosten für die Beantwortung von Kundenanfragen - sind variabel. Ihre Höhe ist somit von der Art und dem Umfang der Kundennachfrage abhängig.

Logistikkosten bilden beim elektronischen Handel von physischen Gütern einen großen Teil der variablen Kosten. Sie sind besonders hoch bei Gütern, die hohe Anforderungen an die Kommissionierung [ $\left.\mathrm{Käm}^{+} 01\right]$, Verpackung und den Transport stellen - wie zum Beispiel Lebensmittel. Dabei stellen die Kosten nicht nur für reine Online-Händler aufgrund von mangelnder Erfahrung und fehlenden Größenvorteilen ein Problem dar $\left[\mathrm{Ba}^{+} 00\right.$, S. 100], sondern auch für stationäre Händler, die für die Abwicklung der Online-Bestellungen eine eigene Logistik benötigen, weil ihre Logistikinfrastruktur auf die Belieferung von Filialen und nicht von Endkunden ausgerichtet ist [Kla00, S. 138]. Hinzu kommt, dass die Kunden in der Regel nicht bereit sind, die hohen Versandkosten zu tragen. Zwar sparen sie durch die Lieferung nach Hause eigene Aufwendungen für Auswahl und Transport der Produkte, nehmen aber diese Einsparungen nicht wahr [Yr01, S. 747-751]. Sie erwarten, dass die Anbieter einen Teil der Lieferkosten tragen oder ganz übernehmen [SchrZi02]. Dies kann im Einzelfall dazu führen, dass die Einnahmen die variablen Kosten nicht decken $\left[\mathrm{Ba}^{+} 00\right.$, S. 100]. Hier wird durch jede Bestellung beim Anbieter Wert vernichtet. 
Weil die meisten Konsumenten das Internet nur in der Informationsphase nutzen, stehen den Investitionskosten und Betriebskosten der Anbieter nur geringe Erlöse aus Online-Verkäufen gegenüber. Selbst durch die Zunahme der Nutzer können die Anbieter nur begrenzt Vorteile erzielen. So steigen zum Beispiel die Kosten, weil für die Bewältigung der gestiegenen Nutzung zusätzliche Rechnerleistung erforderlich ist $\left[\mathrm{Ba}^{+} 00, \mathrm{~S}\right.$. 102]. Auch bei Anbietern, die einerseits für die Erbringung von Zusatzleistungen einen hohen Personaleinsatz benötigen und andererseits physische Güter anbieten, die spezielle Anforderungen an die Logistik stellen [Ha01], ist die Zunahme der Kosten durch zusätzliche Bestellungen besonders hoch. In diesen Fällen werden folglich nur geringe Skaleneffekte generiert.

\subsection{Zukunft des Online-Kanals}

Die aktuelle Situation stellt für viele traditionelle Händler, die im Internet präsent sind, ein Entscheidungsproblem dar. Sie besitzen zwar einen bekannten und von den Kunden akzeptierten Online-Shop, doch ihr Online-Kanal ist unrentabel. Hinzu kommt, dass die möglichen Kostensenkungspotentiale bei vielen Anbietern schon weitgehend ausgeschöpft sind. Für diejenigen, die den Online-Kanal noch nicht vollständig mit ihren stationären Vertriebskanälen vernetzt haben, bedeutet eine stärkere Integration zusätzliche Kosten. Hier müssen die Anbieter nun entscheiden, ob sie ihren Online-Shop ganz oder teilweise weiterführen oder ihre Internet-Präsenz auf reine Imageseiten reduzieren.

Berücksichtigen Unternehmen bei dieser Entscheidung allein die Kosten und Erlöse des Online-Kanals, riskieren sie, eine falsche Entscheidung zu treffen [BöHe01, S. 46]. Denn auch wenn der Online-Kanal isoliert betrachtet unrentabel ist, kann er aus Sicht des gesamten Unternehmens erfolgreich sein. Wie in Kapitel 2.2 bereits beschrieben, gehen vom Online-Kanal positive Wirkungen auf andere Vertriebskanäle und das gesamte Unternehmen aus. Somit ist der Online-Kanal rentabel, wenn sämtliche positiven Wirkungen im Unternehmen höher sind als seine Kosten. Dies kann auch der Fall sein, wenn im Online-Kanal selbst Verluste erzielt werden.

Die Bestimmung der positiven Wirkungen stellt für das Controlling eine Herausforderung dar, da es sich meist um qualitative Wirkungen handelt. Außerdem bleiben Kunden, die sich im Internet nur informieren, häufig anonym. Dasselbe gilt für die Kunden im stationären Handel. Aus diesen Gründen sind die Bewertung der Wirkungen und ihre Zurechnung zum Verursacher komplex, wie die folgenden beiden Beispiele deutlich machen:

- Ein anonymer Kunde findet über die Website die Adresse einer Filiale und gibt dort 200 Euro in bar aus.

- Mit Hilfe der Funktion Produktvergleich der Website trifft ein Kunde eine Produktwahl für ein komplexes technisches Gerät. In einer stationären Filiale 
lässt er sich später nur noch kurz die Handhabung zeigen, bevor er das Produkt kauft. Deshalb nimmt er nur zehn Prozent der durchschnittlichen Beratungszeit in dieser Produktkategorie in Anspruch.

Befindet sich ein Unternehmen in einem frühen Stadium der Vernetzung des Internets mit den bestehenden Vertriebskanälen, kann zur Entscheidung, ob diese intensiviert werden soll, die Optionspreismethode [Sti99; Ho00; HoLeh01; Meh01] herangezogen werden. Diese Methode legt die Annahme zu Grunde, dass die Vernetzung phasenweise erfolgt, wobei nach jeder Phase erneut über die Art der Projektführung entschieden wird. Mögliche Alternativen für ein Unternehmen ohne Hybridstrategien sind die sofortige vollständige Integration, ein Aufschub des Projekts, die Beschränkung der Integration auf einen Bereich oder sogar der Verzicht auf Integration [Me01, S. 3].

\subsection{E-Controlling}

Betrachtet man die Literatur zur Wirtschaftlichkeitsanalyse des Online-Kanals, so finden sich - häufig unter dem Stichwort E-Controlling - in vielen Fällen nur isolierte Betrachtungen des Vertriebskanals Internet oder Untersuchungen reiner Online-Händler [z.B. Erb01; Rie00; FrOe02]. Dabei haben sich die Methoden der Erfolgskontrolle in den letzten Jahren weiterentwickelt. Zu Beginn ihres InternetEngagements haben viele Unternehmen lediglich einfache Nutzungsstatistiken auf der Basis von Logfiles erstellt wie beispielsweise Page Views oder Visits. Mittlerweile haben die meisten Unternehmen begonnen, die Wirtschaftlichkeit des Online-Kanals zu messen [KnuSch00, S. 32; FrOe02, S.179-180]. Hierzu werden unter anderem Finanzkennzahlen und spezielle Formen der Balanced Scorecard [Fr00, S. 227-230; FrOe02, S. 180-186] eingesetzt.

Angesichts der Tatsache, dass die meisten Anbieter im Internet Mehrkanalunternehmen sind, ist die Konzentration auf die isolierte Betrachtung des OnlineKanals überraschend. Die kanalübergreifenden Wirkungen des Online-Kanals werden nur von wenigen Autoren berücksichtigt.

Marr et al. schlagen zahlreiche Messgrößen für die Erfolgsmessung im Electronic Commerce vor. Speziell für Unternehmen mit Hybridstrategien empfehlen sie die Größen „sales growth/decline through each channel“, „,margin trend through each channel“ und „level of existing customer conversions to new channel“ $\left[\mathrm{Ma}^{+} 01, \mathrm{~S}\right.$. 19].

Nach Schwanitz et al. besteht die Wertschöpfung eines Vertriebskanals aus dem direkten Vertriebserfolg, dem indirekten Vertriebserfolg sowie der Kundengewinnung und Kundenbindung [ $\mathrm{Schw}^{+} 02$, S. 30-32]. Indirekte Vertriebserfolge beruhen zum Beispiel auf der Information der Kunden oder der Abschlussanbahnung. Sie betonen jedoch, dass die Ermittlung der Erfolgsanteile der einzelnen Vertriebskanäle nicht einfach ist. Die Aufteilung wird zusätzlich dadurch er- 
schwert, dass einzelne Vertriebskanäle versuchen, den Erfolg ganz für sich zu beanspruchen. Allerdings beschränken sie ihre Betrachtung auf die hybriden Geschäftsprozesse von Banken. Da bei Banken Kunden während ihrer Transaktionen in den stationären Vertriebskanälen nicht anonym bleiben und alle Kundenaktvitäten dokumentiert werden, stehen hier für die Analyse ausreichend Daten zur Verfügung [Schw01].

Böning-Spohr und Hess untersuchen die Wirkungen des Online-Kanals in Medienunternehmen [BöHe01]. Für eine umfassende Wirtschaftlichkeitsbetrachtung müssen auch die Wirkungen des Online-Angebots auf das klassische Angebot untersucht und diese Wirkungen verursachungsgerecht dem Online-Angebot zugerechnet werden. Die Zurechnung kann auf Basis von Nutzeffektketten erfolgen [BöHe01, S. 46-47]. Diese Methode wird im folgenden Kapitel vorgestellt und auf Unternehmen im Konsumgüterhandel angewendet.

\section{Wirtschaftlichkeitsanalyse mit Nutzeffektketten}

Bei der Untersuchung der Wirtschaftlichkeit des Online-Kanals werden die vom Online-Kanal verursachten Kosten mit dem in monetärer Form vorliegenden Nutzen verglichen. Die Kosten setzen sich aus mehreren Kostenkomponenten zusammen, sind aber einfach zu ermitteln und werden im Folgenden zuerst dargestellt. Dagegen ist der Nutzen des Online-Kanals aufgrund der verschiedenen Wirkungsbereiche und vieler qualitativer Nutzenbestandteile schwerer zu ermitteln. Zur Bewertung des Nutzens werden Nutzeffektketten eingesetzt.

\subsection{Kosten des Vertriebskanals Internet}

Betrachtet man die Situation eines Unternehmens mit bestehendem Online-Kanal, so sind die Planungs- und Investitionskosten [Bru97, S. 75-76] als sunk costs für die Entscheidung über den zukünftigen Umfang des Internet-Angebots nicht mehr relevant. Neben den Anlaufkosten fallen aber während der gesamten Nutzungsdauer des Online-Angebots Betriebskosten an, die häufig unterschätzt werden [MoRai03, S. 68]. Die wichtigsten Aufwendungen für den Betrieb des OnlineKanals entstehen in den folgenden Bereichen [Bru97, S. 75-77; Reng00, S. 238239]:

- Bereitstellung: Aufwendungen in Form von Gebühren für Service-Provider

- Wartung: Aufwendungen im Rahmen der Umsetzung technischer Neuerungen, z. B. neue Sicherheitsstandards

- Aktualisierung: Aufwendungen, die durch die Aktualisierung der Website entstehen 
- Vermarktung: Aufwendungen für die Bewerbung des Angebots wie z. B. die Schaltung von Werbebannern, Werbung in stationären Vertriebskanälen oder in traditionellen Medien

- Abwicklung und Logistik: Aufwendungen für die Abwicklung von Transaktionen

- Betreuung: Aufwendungen im Zusammenhang mit der Betreuung von Kunden (z. B. Beantwortung von Kundenanfragen, Betreuung von Foren)

- Kontrolle: Aufwendungen für die Datenerhebung, Datenauswertung und Datenpräsentation

\subsection{Ermitteln der Nutzeffekte}

\subsubsection{Nutzeffektketten}

Die Probleme der Bewertung der positiven Wirkungen des Einsatzes von Informationstechnologie auf andere Unternehmensbereiche hat Schumann bereits im $\mathrm{Zu}-$ sammenhang mit integrierten Informationssystemen beschrieben [Schu92]. Zur Beurteilung ihrer Rentabilität müssen Kosten und Nutzen verglichen werden, wobei die Schwierigkeit darin liegt, den gesamten Nutzen der Informationsverarbeitung zu erfassen [Schu92, S. 1-2]. Denn viele positive Effekte - Nutzeffekte genannt - wirken in unterschiedlichen Bereichen des Unternehmens und müssen zunächst ermittelt werden. Um die Wirkungsbereiche der Informationsverarbeitung zu systematisieren, unterscheidet Schumann Nutzeffekte auf Arbeitsplatzebene, Abteilungsebene, Unternehmensebene und Marktebene [Schu92, S. 63-66].

Die einzelnen Nutzeffekte können in mehrstufigen Ursache-Wirkungs-Beziehungen anordnet werden [Schu92, S. 88ff.; Lin95, S. 35-37; KuTeu99, S. 117; BöHe01, S. 46-47]. Diese Nutzeffektketten werden graphisch durch Knoten und Kanten dargestellt. Die Knoten repräsentieren einzelne Merkmale, die Kanten stellen die Wirkungsrichtung dar. Dabei können die Nutzeffekte den Wirkungsarten Kosten, Produktivität, Qualität, Flexibilität und Wettbewerb zugeordnet werden [Lin95, S. 34-35].

\subsubsection{Nutzeffekte des Vertriebskanals Internet}

Böhning-Spor und Hess unterscheiden drei Wirkungsbereiche eines Online-Angebots [BöHe01, S. 46]:

- Der intramediale Wirkungsbereich umfasst die direkten Wirkungen des Online-Kanals. Das sind zum einen die Kosten für die Bereitstellung des Online-Angebots und zum anderen die Erlöse aus dem Verkauf von Produkten 
und Dienstleistungen sowie weitere Erlöse, die beispielsweise aus der Schaltung von Werbebannern resultieren.

- Der intermediale Wirkungsbereich beinhaltet alle indirekten Wirkungen des Online-Kanals auf die anderen Vertriebskanäle. Ein intermedialer Nutzeffekt tritt zum Beispiel ein, wenn Kunden über die Website auf den stationären Vertriebskanal eines Anbieters aufmerksam werden und infolgedessen im stationären Handel einkaufen.

- Der unternehmensbezogene Wirkungsbereich umfasst alle Wirkungen, die sich auf das gesamte Unternehmen beziehen. Zum Beispiel wirkt sich der Imagegewinn eines erfolgreichen Internet-Auftritts auf alle Vertriebskanäle eines Unternehmens positiv aus.

In Abbildung 2 sind die drei Wirkungsbereiche dargestellt.

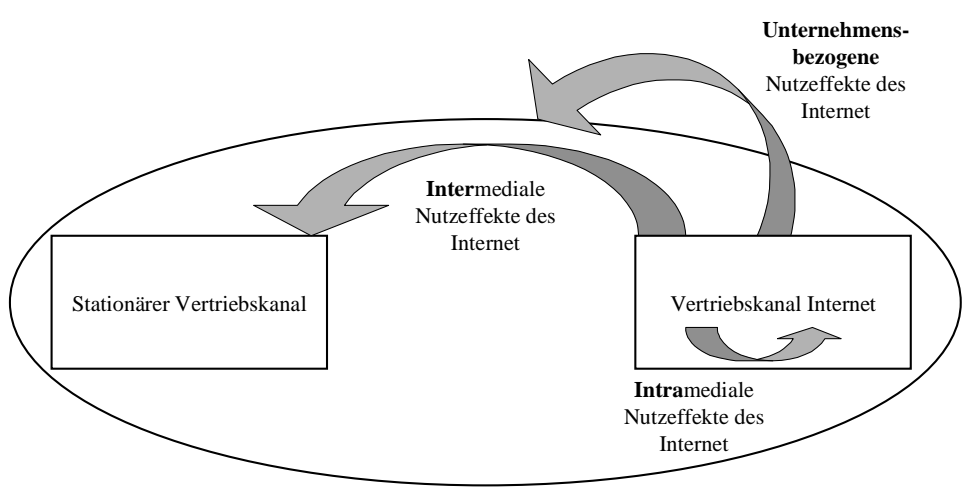

Abbildung 2: Intramediale und intermediale Nutzeffekte [in Anlehnung an BöHe01, S. 46]

Hinsichtlich der Bewertbarkeit ist festzustellen, dass die intramedialen Nutzeffekte in der Regel monetär und somit leicht zu erfassen sind. Schwerer zu bewerten, weil größtenteils nicht direkt monetär bewertbar sind die intermedialen und unternehmensbezogenen Nutzeffekte, wobei Letztere besonders schwierig zu erfassen sind [BöHe02, S. 108]. Im Folgenden werden deshalb nur die intermedialen Nutzeffekte des Online-Kanals auf den stationären Vertriebskanal untersucht. Jedoch können auch hier aufgrund des großen Umfangs und der Vielschichtigkeit der Wirkungen nur einige ausgewählte Wirkungen dargestellt werden.

Um Nutzeffektketten für die intermedialen Effekte des Interneteinsatzes herzuleiten, wurden aus der Beschreibung der Vorteile des Online-Kanals für die stationären Vertriebskanäle in Kapitel 2.2.2 zunächst die positiven Wirkungen der Vernetzung ermittelt. In einem zweiten Schritt wurden dann die unterschiedlichen 
Nutzeffekte entsprechend ihrer Ursache-Wirkungs-Beziehungen in mehrere Nutzeffektketten angeordnet (siehe Abbildung 3).

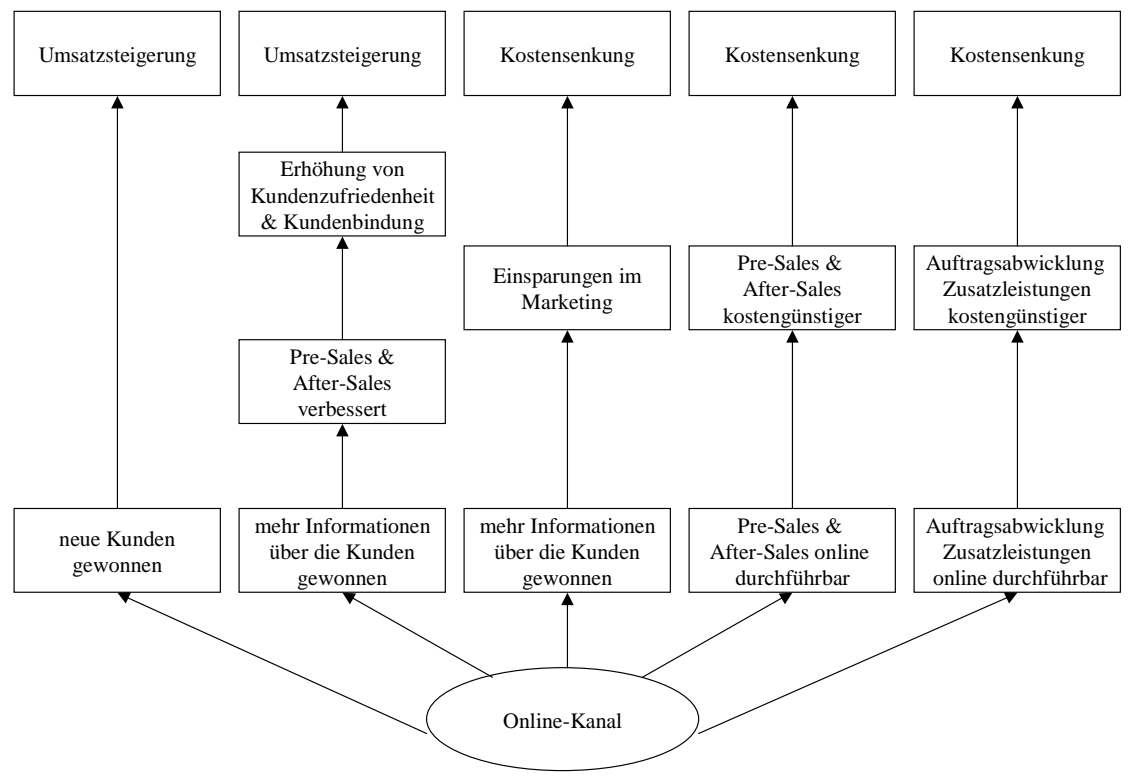

Abbildung 3: Nutzeffektketten des Vertriebskanals Internet

Die einzelnen Nutzeffekte stellen sich wie folgt dar:

- Da die Kunden aufgrund ihrer Unsicherheit zwar das Internet zur Information nutzen, anschließend aber in traditionellen Vertriebskanälen kaufen, können durch die Präsenz im Internet neue Kunden gewonnen und somit der Umsatz gesteigert werden. Hierzu sollten im Internet Informationen über die physische Präsenz leicht auffindbar sein.

- Aus den Bestelldaten des Online-Shops können Informationen über Kunden gewonnen werden. Weitere Informationen über Kunden können durch die Pflicht der Registrierung vor der Inanspruchnahme von Service-Leistungen gewonnen werden.

- Diese Informationen können verwendet werden, um Angebot stärker an die Präferenzen der Kunden anzupassen, wodurch sich für die Kunden des stationären Geschäfts besonders Verbesserungen bei den Pre-Sales- und AfterSales-Leistungen ergeben. Viele Kunden informieren sich heute vor dem Kauf im Internet, erwerben ihre Produkte aber im stationären Handel, dem dieser Service somit hauptsächlich zu Gute kommt. Hier finden sie umfangreichere Informationen und neue Dienstleistungen wie den Vergleich von Produkten. Auch nach dem Kauf können durch die Internet-Präsenz bessere Leistungen 
erbracht werden. Zum Beispiel können bei Problemen FAQs genutzt oder der Kundendienst per E-Mail kontaktiert werden.

Der Service kann sich für die Kunden nicht nur dann verbessern, wenn sie unterstützend das Internet nutzen. Auch die Beratungsqualität des Verkaufspersonals kann durch die schnellere Informationsbereitstellung und bessere Verfügbarkeit von Informationen durch das Online-Angebot gesteigert werden, indem die Website auch dem Verkaufpersonal als Informationsquelle zur Verfügung steht.

- Die durch den Online-Kanal induzierten Vorteile wie die Verbesserung des Service sowie die Vereinfachung und Beschleunigung der Kommunikation erhöhen die Kundenzufriedenheit und Kundenbindung und damit auch den Umsatz der stationären Vertriebskanäle.

- Die im Online-Kanal gewonnenen Informationen können auch genutzt werden, um Einsparungen im Marketing zu erzielen. Zum einen stellt das Internet eine kostengünstige Möglichkeit dar, mit den Kunden zu kommunizieren. Registrierte Kunden können beispielsweise über eine Sonderaktion im stationären Handel per E-Mail informiert werden. Zum anderen werden heute Werbekampagnen beobachtet, die die Ebene der Produktinformation größtenteils auf die Website verlagern, indem in der klassischen Werbung wenig mehr als die Internetadresse des Anbieters vermittelt wird.

- Nutzen die Kunden des stationären Handels den Online-Kanal vor und nach dem Kauf im Geschäft, ergeben sich Einsparungen bei Pre-Sales und AfterSales-Leistungen. Dabei werden vor allem Personalkosten gespart. Besonders hoch fallen diese Einsparungen aus, wenn die bereitgestellten Informationen aus bereits in elektronischer Form vorliegenden Quellen generiert werden können. Beispielsweise wenn die Produktinformationen auf der Website eines Händlers vom Hersteller bereitgestellt werden.

- Im Rahmen des After-Sales-Service für Kunden des stationären Geschäfts werden im Internet auch kostenpflichtige Zusatzleistungen wie Ersatzteile, Lizenzverlängerungen oder Softwareupdates angeboten. Diese werden hier isoliert betrachtet, da sie - im Gegensatz zu den für die Kunden kostenlosen Leistungen - Funktionen zur Unterstützung von Bestellung und Bezahlung erfordern. Im Vergleich zu einem stationären After-Sales-Service ist die Auftragsabwicklung bei den Zusatzleistungen günstiger. Insbesondere digitale Güter wie Software und Lizenzen können über das Internet geliefert werden.

\subsection{Bewerten der Nutzeffekte}

Das Ziel der Bewertung ist es, eine monetäre Größe zu ermitteln, die den Nutzen der gesamten Wirkungskette zusammenfasst. Jedoch stellt sich hierbei das Problem, dass die intermedialen Nutzeffekte vielmals nicht monetär bewertbar sind, 
sondern in Form von nicht-monetären quantitativen und qualitativen Wirkungen vorliegen [Lin95, S. 33-34]. In diesem Fall ist eine Bewertung nur dann möglich, wenn die Wirkungen mit Hilfe der Nutzeffektketten indirekt monetarisiert werden können [Schu92, S. 218ff; Lin95, S. 100]. Dabei werden beispielsweise quantitative Effekte in monetäre Wirkungen überführt indem z.B. Arbeitszeiteinsparungen mit dem Stundensatz bewertet werden. Es gibt aber auch Wirkungsketten bei denen der Zusammenhang zwischen den Effekten komplexer und somit schwer ableitbar ist. In diesen Fällen können die Wirkungen lediglich geschätzt oder nur verbal beschrieben werden [Lin95, S. 103-106].

Indem nun die einzelnen Nutzeffekte entlang der verschiedenen Wirkungsketten bewertet werden, ergibt sich für jede Kette ein Endeffekt, der den Nutzen des gesamten Strangs darstellt. Der gesamte intermediale Effekt des Online-Kanals ergibt sich deshalb aus der Aggregation aller Endeffekte [Lin95, S.106-109].

Ein großes Problem bei der Bewertung der intermedialen Nutzeffekte des OnlineKanals stellt die Verfügbarkeit der erforderlichen Daten dar. Denn im Bereich der Konsumgüter bleiben die Kunden beim Kauf im stationären Handel in der Regel anonym. Kundendaten können nur durch Kartenzahlungen, Kreditgewährung, Lieferungen oder die Verwendung von Kundenkarten gewonnen werden. Nutzen die Kunden das Internet ausschließlich zur Information, so können sie auch hier anonym bleiben. Der Anbieter hat in diesem Fall lediglich die Möglichkeit, durch Serverprotokolle und Cookies Informationen über das Verhalten der Kunden zu generieren. Aus diesem Grund versuchen viele Anbieter die Kunden mit anmeldepflichtigen Zusatzleistungen zur Registrierung zu bewegen. Anders ist dagegen die Situation, wenn Kunden die Möglichkeiten zur Interaktion und Kommunikation nutzen. Dann stehen dem Unternehmen Daten aus Suchanfragen, Formulareinträgen oder E-Mails zur Verfügung [Reng00, S. 241; LiSch01, S.74-75]. Haben Kunden sogar im Internet bestellt, so verfügt das Unternehmen über umfangreiche Kundeninformationen.

Für die beschriebenen Nutzeffektketten soll im Folgenden ein Bewertungsansatz skizziert werden:

- Um das Ausmaß der Umsatzsteigerungen durch die Gewinnung neuer Kunden für das stationäre Geschäft zu ermitteln, werden Informationen über die Veränderung der Anzahl der Kunden, des Umsatzes und der Marktanteile benötigt. Bei der Analyse der Kundenstruktur sind mögliche Verschiebungen durch die Einführung eines neuen Vertriebskanals zu berücksichtigen [Bru97, S. 131135]. Zum Beispiel können Kunden des stationären Handels zum OnlineKanal wechseln. Der Nutzeffekt ,neue Kunden gewonnen“ kann monetarisiert werden, indem die Zahl der zusätzlichen Kunden mit den erwarteten Nettozahlungen bewertet werden.

Allerdings ist es in der Praxis meistens schwierig zu ermitteln, welche Kunden aufgrund des Online-Kanals neu gewonnen wurden. Hier können Kundenbefragungen eingesetzt werden. Auch Gutscheine, die im Online-Kanal bezogen 
sowie zu Hause ausgedruckt werden können und in den stationären Filialen einlösbar sind, dienen der Informationsgewinnung. Einen weiteren Anhaltspunkt darüber, wie viele Kunden durch die Internet-Präsenz auf das stationäre Geschäft aufmerksam werden, geben Informationen über die Häufigkeit, mit der im Internet Informationen über die Standorte des stationären Handels aufgerufen werden.

- Eine weitere Wirkungskette beruht darauf, dass im Online-Kanal umfangreiche Informationen über Kundenpräferenzen gewonnen werden können. Auf der Basis dieser Informationen können die Aktivitäten im Pre- und After-SalesBereich stärker an die Kundenbedürfnisse angepasst werden, was die Kundenzufriedenheit und Kundenbindung erhöht. Dies hat für das Unternehmen den positiven Effekt, dass weniger Kunden zur Konkurrenz abwandern.

Allerdings ist der Wirkungszusammenhang in dieser Nutzeffektkette sehr komplex [MoRai03, S. 74-76] und kann zu großen Teilen nur geschätzt werden. Informationen über die Kundenzufriedenheit können beispielsweise durch Kundenbefragungen gewonnen werden. Ein Vergleich der Kundenzufriedenheit vor und nach Einführung des Online-Kanals gibt Hinweise auf den Beitrag des neuen Vertriebskanals. Im Bezug auf die Nutzeffekte des Online-Angebots sollten sich die Fragen speziell auf die Nutzung diese Angebots durch Kunden des stationären Geschäfts beziehen. Weitere Hinweise liefert die Analyse der Kundenanfragen und Reklamationen (z.B. sind Reklamationen bezüglich des stationärem Geschäfts per E-Mail eingegangen ?).

Der Wert der Kundenbindung kann über die abdiskontierten zukünftigen Nettozahlungen derjenigen Kunden, die aufgrund der Existenz des Online-Kanals nicht zur Konkurrenz abwandern, operationalisiert werden [Bru97, S. 128129].

- Zusätzliche Informationen über Kunden, die im Online-Kanal gewonnen werden, können im Marketing dazu verwendet werden, Aktionen zielgerichteter zu gestalten und damit Kosteneinsparungen zu erzielen. Eine mögliche Messgröße ist die Anzahl der gewonnen Kundenprofile. Da in der Regel nur Kunden, die in der Nähe einer Filiale wohnen von den Vorteilen einer Hybridstrategie profitieren können, ist die Anzahl entsprechend zu gewichten. Dabei muss berücksichtigt werden, dass die „Reichweite der Hybridstrategie“ von unterschiedlichen Faktoren abhängig ist. Zum Beispiel nehmen Kunden für Nischenprodukte deutlich längere Wege in Kauf. Die Monetarisierung dieser Wirkungen ist aufgrund der Komplexität der Zusammenhänge häufig nicht einfach.

- Um die Einsparungen bei Pre-Sales und After-Sales-Leistungen zu ermitteln, müssen die über die Website erbrachten Serviceleistungen festgehalten werden. Dies kann zum Beispiel über die Page Views der Informationsseiten, die Anzahl der Downloads von Produktinformationen oder die Anzahl der E-MailAnfragen erfolgen. Zur Ermittlung der Gesamthöhe der Einsparungen muss zusätzlich die Anzahl der Kunden des stationären Geschäfts ermittelt werden, 
die zwar in der Pre-Sales- und After-Sales-Phase den Online-Kanal nutzen, ihre Einkäufe aber in den stationären Vertriebskanälen tätigen.

- Die Einsparungen bei der Auftragsabwicklung von Zusatzleistungen können durch Kostenvergleich der Geschäftsprozesse im stationären Handel und im Internet ermittelt werden [MoRai03, S. 67-68 u. 78-79].

\section{Zusammenfassung und Ausblick}

Durch die Berücksichtigung der intermedialen Nutzeffekte des Internets stellt sich die Rentabilität des Online-Kanals in einem anderen Licht dar, denn traditionelle Unternehmen können mit Hilfe von Hybridstrategien Vorteile für ihre stationären Vertriebskanäle erzielen. Als Instrument für die Wirtschaftlichkeitsanalyse des Online-Kanals in Mehrkanalunternehmen können Nutzeffektketten eingesetzt werden. Sie stellen dar, welche Ursachen und Wirkungen die Gewinne im stationären Handel positiv beeinflussen und bilden die Grundlage für die Monetarisierung qualitativer Effekte.

Allerdings müssen auch die Grenzen der Analyse anhand von Nutzeffektketten im Auge behalten werden [BöHe01, S. 47]. Für die Aufstellung der Ursache-Wirkungs-Beziehungen gibt es keine eindeutigen Vorgaben und somit ist das Ergebnis von der subjektiven Einschätzung des Bewertenden abhängig. Weiterhin können qualitative Effekte nicht immer mit Hilfe von Ursache-Wirkungs-Ketten monetarisiert werden. Bei positiven Wirkungen auf das gesamte Unternehmen können die Wirkungen nur schwer nachvollzogen werden und sind deshalb kaum mit Nutzeffektketten darstellbar.

Wie der Literaturüberblick in Kapitel 3.3 gezeigt hat, gibt es wenige Ansätze zur Erfassung und Bewertung intermedialer Nutzeffekte des Online-Kanals in Mehrkanalunternehmen. Hier besteht Bedarf zu weiterer Forschung. Neben der Entwicklung geeigneter Analyseinstrumente, werden auch weitere Verfahren zur Gewinnung von kanalübergreifenden Kundendaten benötigt.

\section{Literatur}

[Arm02] Armbruster, K.: Hybrid Strategies in Consumer Retail Markets. In: Proceedings of the Ninth Research Symposium on Emerging Electronic Markets (RSEEM 2002). Institute of Business Economics (IAB), University of Applied Sciences (FHBB), Basel 2002, S. 1-10.

[ArmSch02] Armbruster, K.; Schober, F.: Hybridstrategien im Multikanal-Vertrieb. In: WiSt 31, 2002, H. 6: S. 347-350. 
$\left[\mathrm{Ba}^{+} 00\right]$ Barsh, J.; Crawford, B.; Grosso, Ch.: How e-tailing can rise from the ashes. The McKinsey Quarterly, 2000, H. 3: S. 98-109.

[BöHe01] Böning-Spohr, P.; Hess, Th.: Zum Veränderungsbedarf im Verlagscontrolling. In: Weber, J.; Schumann, M. (Hrsg.) E-Business \& Controlling, Kostenrechnungspraxis Sonderheft 2, 2001, S. 40-51.

[BöHe02] Böning-Spohr, P.; Hess, Th.: Analyse der Wechselwirkungen zwischen Printund Online-Angeboten mittels Wirkungsketten. In: Fantapié Artobelli, C. (Hrsg.) Print contra Online? Verlage im Internetzeitalter. Verl. R. Fischer: München, 2002, S. 103112.

[Bru97] Bruhn, M.: Multimedia-Kommunikation: systematische Planung und Umsetzung eines interaktiven Marketing-Instruments. Beck: München, 1997.

[Bu ${ }^{+}$97] Bucklin, Ch. B.; Thomas-Graham, P. A.; Webster, E. A.: Channel Conflict: When is it dangerous? The McKinsey Quarterly, 1997, H. 3: S. 36-43.

[Erb01] Erben, R. F.: e-controlling - Anforderungen an das Controlling im e-business. Kostenrechnungspraxis 45,2001 , H. 4: 235-241.

[Fr00] Fröhling, O.: Controlling in der New Economy: Konzeption und Umsetzung von EIntelligence. Kostenrechnungspraxis 44, 2000, H. 4: 223-231.

[FrOe02] Fröhling, O.; Oehler, K.: E-Business braucht E-Controlling. Controlling 14, 2002, H. 3: $179-188$.

[GeSch99] Gebauer, J.; Scharl, A.: Between Flexibility and Automation: An Evaluation of Web Technology from a Business Process Perspective. Journal of Computer-Mediated Communication 5, 1999, H. 2.

[Goe02] Goersch, D.: Multi-Channel Integration and its Implications for Retail Web Sites. In: Proceedings of the $10^{\text {th }}$ European Conference on Information Systems, June 6-8 2002, Gdansk, S. 748-758.

[GuGa00] Gulati, R.; Garino, J.: Get the Right Mix of Bricks \& Clicks. Harvard Business

Review 78, 2000, H. 3: S. 107-114.

[Ha01] Hallowell, R.: "Scalability": the paradox of human resources in e-commerce. International Journal of Service Industry Management 12, 2001, H. 1: S. 34-43.

[Ho00] Hommel, U.: Bewertung der "New Economy" auf Realoptionsbasis. WertpapierMitteilungen - Zeitschrift für Wirtschafts- und Bankrecht 31, 2000, H. 5: S. 1535-1536.

[HoLeh01] Hommel, U.; Lehmann, H.: Einsatzmöglichkeiten des Realoptionsansatzes in der New Economy aus Controlling-Sicht. Controlling 13, 2001, H. 1: S. 15-21.

[Käm $\left.{ }^{+} 01\right]$ Kämäräinen, V.; Smaros, J.; Jaakola, T.; Holmström, J.: Cost-effectiveness in the e-grocery business. International Journal of Retail \& Distribution Management 29, 2001: 41-48.

[Kla00] Klaus, P.: Zum "materiellen Internet": Herausforderungen an die Logistik. In: Scheffler, W.; Voigt, K.-I. (Hrsg.): Entwicklungsperspektiven im Electronic Business. Gabler: Wiesbaden, 2000, S. 133-151. 
[KnuSch00] Knust, P.; Schindera, F.: e-Controlling, Vortrag im Rahmen der Reihe „ECommerce - Wettbewerbsvorteile realisieren" des Zentrums für Europäische Wirtschaftsforschung am 18.10.2000 in Mannheim. http://www.schindera.de/e-controlling/ docs/e-controlling_k nust_schindera_181000.pdf, Abruf am 2003-01-14.

[KuTeu99] Kurbel, K.; Teuteberg, F.: Umsatzsteigerungen durch Internet-Engagement?. In: Scheer, A.-W.; Nüttgens, M. (Hrsg.) Electronic Business Engineering, 4. Internationale Tagung Wirtschaftsinformatik 1999. Physica-Verl.: Heidelberg, 1999, S. 113-134.

[La94] Langenohl, T.: Systemarchitekturen elektronischer Märkte. Dissertation, Hochschule St. Gallen, 1994

[LiSch01] Link, J.; Schmidt, S.: E-Business und Marketing-Controlling. In: Weber, J.; Schumann, M. (Hrsg.) E-Business \& Controlling, Kostenrechnungspraxis Sonderheft 2, 2001, S. 73-80.

[Lin95] Linß, H.: Integrationsabhängige Nutzeffekte der Informationsverarbeitung - Vorgehensmodell und empirische Ergebnisse. Gabler: Wiesbaden, 1995.

[Ma ${ }^{+}$01] Marr, B.; Neely, A.; Adams, Ch.: Measuring and Managing Performance in eBusiness. In: Weber, J.; Schumann, M. (Hrsg.) E-Business \& Controlling, Kostenrechnungspraxis Sonderheft 2, 2001, S. 12-20.

[Me01] Mehler-Bicher, A.: Bewertung von e-Business Investitionen mithilfe von praxisadäquater Optionspreismodelle. Arbeitsbericht, Fachhochschule Mainz, 07/2001. http://www.competence-site.de, Abruf am 2003-01-28.

[MoRai03] Mogollon, M.; Raisinghani, M.: Measuring ROI in e-Business: A Practical Approach. Information Systems Management 20, 2003, H. 2: S. 63-81.

[Ni $\left.\mathrm{Ni}^{+} 01\right]$ Nicholson, M.; Clarke, I.; Blakemore, M.: Multichannel Consumer Behaviour in the Retail Fashion Sector: Toward a longitudinal ethnography; Paper presented at the $11^{\text {th }}$ International Conference on Research in the Distributive Trades; Tilburg, $27^{\text {th }}-29^{\text {th }}$ June 2001. http://www.dur.ac.uk/mike.nicholson/tilburg_paper.htm, Abruf am 2003-0108 .

[Pi02] Piller, F.: Mass Customization. In: Li, Z.; Possel-Doelken, F. (Hrsg.) Strategic Production Networks. Springer: New York et al, 2002, S. 389-421. http://www.masscustomization.de/download/spn2000.pdf, Abruf am 2003-01-18.

[Reng00] Rengegelshausen, O.: Online-Marketing in deutschen Unternehmen. Gabler: Wiesbaden, 2000.

[Rie00] Rieg, R.: Controlling und E-Business. Controlling 12, 2000, H. 8/9: S. 403-407.

[Ro97] Rohrbach, P.: Interaktives Teleshopping: elektronisches Einkaufen auf dem Informationhighway. Wiesbaden: Deutscher Universitätsverlag, 1997.

[SchmLi98] Schmid, B.; Lindemann, M.: Elements of a Reference Model for Electronic Markets. In: Proceedings of the $31^{\text {st }}$ Annual Hawaii International Conference on Systems Science, Hawaii, January 6-9 1998, Vol. IV, S. 193-201.

[SchoGo02] Schoenbachler, D.; Gordon, G.: Multi-channel shopping: understanding what drives channel choice. Journal of Consumer Marketing 19, 2002, H. 1: S. $42-53$. 
[SchrZi02] Schröder, H.; Zimmermann, G.: Lieferkosten-Modelle im Electronic Retailing Eine Bestandsaufnahme aus Sicht der Anbieter und Nachfrager. In: Ahlert, D.; Olbrich, R.; Schröder, H. (Hrsg.) Electronic Retailing. Dt. Fachverlag: Frankfurt a. M., 2002, S. 337-361.

[Schu92] Schumann, M.: Betriebliche Nutzeffekte und Strategiebeiträge der großintegrierten Informationsverarbeitung. Springer: Berlin et al., 1992.

[Schw01] Schwanitz, J.: Web-Controlling in der Multikanal-Vertriebssteuerung. Die Bank, 2001, H. 8: 589-595.

[Schw $\left.{ }^{+} 02\right]$ Schwanitz, J.; Kipker, I.; Levermann, V.: Vertriebssteuerung und Kostenmanagement. Bankmagazin, 2002, H. 7: S. 30-32.

[St02] Steinfield, C.: Understanding Click and Mortar E-Commerce Approaches: A Conceptual Framework and Research Agenda. Journal of Interactive Advertising. 2, 2002, H. 2. http://www.jiad.org/vol2/no2/steinfield/index.html, Abruf am 2003-01-08.

[StK199] Steinfield, C.; Klein, S.: Local vs. Global Issues in Electronic Commerce. Electronic Markets 9, 1999, H. 2: S. 1-6.

[St ${ }^{+} 99$ ] Steinfield, C., Mahler, A., Bauer, J.: Electronic Commerce and the Local Merchant: Opportunities for Synergy Between Physical and Web Presence. Electronic Markets 9, 1999, H. 2: S. 51-57.

[Sti99] Stickel, E.: Bewertung von IV-Entwicklungsprojekten mit Methoden der Optionspreistheorie. In: Scheer, A.-W.; Nüttgens, M. (Hrsg.) Electronic Business Engineering, 4. Internationale Tagung Wirtschaftsinformatik 1999. Physica-Verl.: Heidelberg, 1999, S. 685-707.

[TaTho00] Tan, Y.-H.; Thoen, W.: An outline of a trust model for electronic commerce. Applied Artificial Intelligence 14, 2000, H. 8: S. 849-862.

[Yr01] Yrjölä, H.: Physical distribution consideration for electronic grocery shopping. International Journal of Physical Distribution \& Logistics 31, 2001: 746-761. 